\title{
Numerical Study of a Wide-angle Near-Perfect Absorber for the Visible Regime Incorporating Metal-Dielectric-Metal Subwavelength Grating Structure
}

\section{Wenlong Zou}

Soochow University

Heng Zhang

Soochow University

Yun Zhou ( $\square$ zyun@suda.edu.cn )

Soochow University https://orcid.org/0000-0001-6388-4331

\section{Research Article}

Keywords: Optical devices, Metamaterial absorber, Visible, Absorptivity

Posted Date: January 10th, 2022

DOI: https://doi.org/10.21203/rs.3.rs-1222186/v1

License: (c) (1) This work is licensed under a Creative Commons Attribution 4.0 International License. Read Full License 


\section{Abstract}

A near-perfect absorber for the visible regime based on metal-dielectric-metal subwavelength grating structure with the refractory metals is designed and demonstrated numerically. The absorber presents an average absorption over $98.4 \%$ in the visible regime at normal incidence. Angle-relative analysis shows that the proposed structure has good angle-tolerance. The high average absorption ( $86.6 \%)$ in the visible region can be maintained with the incident angles up to $60^{\circ}$. Through the analysis of the magnetic field, the physical origin is verified that this excellent absorption performance mainly stems from the cooperative effect of surface plasmonic resonances and the intrinsic broadband spectral responses by the refractory metals. In addition, the dependence of the absorption spectrum of the proposed absorber on the structural parameters is analyzed. This work provides an idea for the design of high-performance absorbers and has potential applications in advanced light energy capture and integration systems.

\section{Introduction}

Since Landy et al. first demonstrated a perfect metamaterial absorber (MA) at $11.5 \mathrm{GHz}$, MAs have attracted great interest because of their wide applications, such as solar cells, plasmonic sensors, photodetectors, and thermal emitters [1-27]. Adjustable-band, omnidirectional, and polarizationindependent absorbers based on the metamaterial or meta-surface have been investigated. Under appropriate conditions, the absorption can be enhanced by intrinsic electron resonance and magnetic resonance, including surface plasmon resonance (SPR), cavity mode resonance (CMR), Mie resonance (MR) or hybrid resonance [5-10]. In the previous studies, a lot of research work focuses on infrared band, or longer band absorption [4-5]. In recent years, with the rapid development of micro-nano processing technology, people gradually pay attention to the absorber working in the visible regime [10, 28-34]. For example, Qinyu Qian proposed and experimentally demonstrated an ultrathin meta-surface perfect absorber based on coupled Mie resonances with dielectric Si nanopillars and metallic Cr nanopillars [10]. Sunwo Han et al. numerically investigated and analyzed the electromagnetic resonances on a 2D tandem grating and its application for broadband absorption in the visible spectrum [28]. Minghui Luo et al. proposed a broadband absorber based on a metallic substrate and a 1D subwavelength metallic grating with the grooves filled with dielectric material in the visible regime [29]. Recent literature shows that the combination of meta-surface and the refractory material can achieve efficient broadband absorption in the visible band. Yuyin Li proposed an ultra-broadband perfect absorber composed of the insulator-metalinsulator grating on the metal-insulator-metal (MIM) film stacks utilizing refractory materials, achieving over $90 \%$ absorption in the wavelength range from $570 \mathrm{~nm}$ to $3539 \mathrm{~nm}$ with an average absorption of $97 \%$ [24]. These structures mentioned above are very sensitive to structural parameters, so there are strict requirements for machining means, which limit practical device applications. Although some progress has been made, further research on broadband absorbers using refractory materials and refractory materials is needed.

In this paper, a wide-angle near-perfect absorber incorporating metal-dielectric-metal subwavelength grating with refractory materials is presented. An average absorption of $98.4 \%$ for the whole visible 
regime at normal incidence is attained. Particularly, near-perfect absorption (about 99.6\%) is achieved at $\lambda=600 \mathrm{~nm}$. Moreover, the high average absorption (86.6\%) can be maintained in the visible region with the incident angle varying from 0 to $60^{\circ}$. The high broadband absorption is attributed to the cooperative effect of surface plasmonic resonances supported by the multi-layered structure and the intrinsic broadband spectral responses by the refractory metals. Additionally, the absorption spectra of different structure parameters are studied. The near-perfect absorber presented in this paper has potential application prospects in color-printing, solar-energy harvesting, and other fields.

\section{Simulation And Discussion}

Figure 1 shows the schematic diagram of the proposed absorber, consisting of the bottom metal layer of the refractory material tungsten $(\mathrm{W})$, the dielectric layer ( $\mathrm{SiO} 2)$, and the subwavelength metal $(\mathrm{W})$ grating layer. The heights of them are $h_{1}, h_{2}$, and $d$, respectively. The metal grating in this structure is also characterized by the period $p$, the ridge width $w$, and the duty cycle $F(F=w / p)$. The refractive index of $W$ is fitted by the Drude-Lorentz model [35]. The refractive index of the dielectric layer is 1.5. In the structure, the bottom $\mathrm{W}$ layer with the thickness larger than the skin depth is employed to hinder the transmission. The subwavelength metal grating arrays are symmetric along the $X$ and $Y$ directions, indicating that the desirable polarization-independence could be deservedly obtained. In order to simplify, the TM-polarized light incident from the top air side at an angle of $\theta$ is selected for analysis. The simulated absorption (A), reflection (R) and transmission $(T)$ is performed using the rigorous coupled analysis (RCWA) method [36-37].

Figure 2 shows the simulated $\mathrm{R}, \mathrm{T}$ and $\mathrm{A}$ at normal incidence for the visible region, respectively, where $p=280 \mathrm{~nm}, \mathrm{~F}=0.5, \mathrm{~h}_{1}=80 \mathrm{~nm}, \mathrm{~h}_{2}=50 \mathrm{~nm}$, and $\mathrm{d}=30 \mathrm{~nm}$. As shown in Fig. 2, the average absorption is about $98.4 \%$ from $400 \mathrm{~nm}$ to $700 \mathrm{~nm}$ and the absorption peak $(A \approx 99.6 \%)$ appears at the wavelength of $\lambda=$ $600 \mathrm{~nm}$. Figure 3 shows the absorption spectra for TM-polarized light in the visible region with the incident angle varying from $0^{\circ}$ to $60^{\circ}$. The average absorption is still over $86.6 \%$ when the incident angle adds up to $60^{\circ}$. The above simulation results show that the proposed absorber has robust angle tolerance, which is crucial and needful for the applications in color-printing and harvesting solar energy.

To understand the physical mechanism of the broadband absorption of the proposed absorber, the magnetic field at the absorption peak $(\lambda=600 \mathrm{~nm})$ and several non-absorption peak positions $(\lambda=400$ $\mathrm{nm}, 500 \mathrm{~nm}$, and $700 \mathrm{~nm}$ ) is presented in Fig. 4. From Fig. 4, it is found that the magnetic field is mainly confined within the multi-layered structure at these wavelengths. As can be seen from Fig. 4(a), at $\lambda=400$ $\mathrm{nm}$, the magnetic field is mainly confined at the interface between the bottom metal layer and the dielectric layer, just below the metal grating groove, indicating the excitation of local surface plasmon resonance (SPRs) $[24,38]$. At $\lambda=500 \mathrm{~nm}, \lambda=600 \mathrm{~nm}$ and $\lambda=700 \mathrm{~nm}$, the effect of the localized SPRs at the interface between the bottom metal layer and the dielectric layer underly the metal grating groove is weakened. However, the enhanced magnetic field is mainly located at the region confined by the periodic subwavelength grating ridges and the bottom metal layer. In this case, the SPRs excitation on the lower 
surface of the subwavelength metal grating and the upper surface of the bottom metal layer and their coupling result in strong absorption [24,38].

To further analyze the physical mechanism of the proposed absorber, the Poynting vector distributions at $\lambda=400 \mathrm{~nm}$ (non-absorption peak) and $\lambda=600 \mathrm{~nm}$ (absorption peak) for TM-polarized light is presented in Fig. 5. At $\lambda=400 \mathrm{~nm}$, most of the incident energy flow passes through the grooves of the metal grating into the bottom metal layer, and a small amount of energy flows into the area below the metal grating ridges. At $\lambda=600 \mathrm{~nm}$, most of the incident energy flows into the area below the metal grating ridges, and part of them flows through this area and then into the bottom metal layer. Finally, the energy will be dissipated by the Ohmic losses of $\mathrm{W}$. Therefore, the designed structure can permit high polarizationindependent absorption in the visible regime. Most of the energy is found to be trapped in the multilayered structure, which gives a solid proof that the SPRs play a key role in the absorptive behavior in the structure.

The dependence of the absorption spectra of the TM-polarized light on the period ( $p)$, the height (d), and the duty cycle (F) of the subwavelength metal grating are demonstrated in Fig. 6 (a), Fig. 6 (b), and Fig. 6 (c), respectively. As can be seen from Fig. 6 (a), when the period varies from $250 \mathrm{~nm}$ to $350 \mathrm{~nm}$, the absorption in the whole visible band is about $98.0 \%$. From Fig. 6 (b), it is indicated that the broadband character is also controlled by $d$. If $d$ is too large $(>60 \mathrm{~nm})$, the reflection of W will be obvious, leading to a decrease in absorption efficiency. When $\mathrm{F}$ varies from 0.4 to 0.6 , the proposed structure absorbs efficiently in the whole visible band, as shown in Fig. 6 (c). From the production feasibility point of view, the best parameter for wide bandwidth is $F=0.5$. The dependence of the absorption spectrum of the TM polarized light on the height $\left(h_{2}\right)$ of the dielectric layer is demonstrated in Fig. $6(d)$. It is obvious that $h_{2}$ is extremely significant for broadband character. When $h_{2}$ is in the range of $20 \mathrm{~nm}-80 \mathrm{~nm}$, the structure has good broadband absorption performance. When $h_{2}$ changes from $80 \mathrm{~nm}$ to $150 \mathrm{~nm}$, the absorption performance decreases sharply, because the coupling of surface plasmon waves decreases. When $h_{2}$ is greater than $150 \mathrm{~nm}$, it shows the characteristics of narrow band absorption. From the above analysis, it can be seen that the designed structure has greater tolerance for structural parameters, so it can reduce the rigor of the process.

The influence of metal materials on the absorption performances of the proposed absorber by replacing the $\mathrm{W}$ layer by $\mathrm{Ti}, \mathrm{Cr}$ and $\mathrm{Al}$ while keeping the geometry parameters constant are analyzed in Fig. 7(a). And the absorption spectrum without the corresponding metal grating layer is shown in Fig. 7(b). It can be inferred from Fig. 7 (a) that when using W, Ti or Cr, the absorption spectrum exhibits superior performance with an broadband perfect absorption (over $90 \%$ ). When using $\mathrm{Al}$, the absorption in the visible region is significantly reduced. The discrepancy observed here is decided by the metal's intrinsic dispersion property. In the proposed structure, for the refractory metal of W, $\mathrm{Ti}$ or $\mathrm{Cr}$ with an appropriate thickness satisfies the impedance match conditions and thus achieves high absorption in the visible range [39]. In Fig. 7(b), it can be seen that when there is no metal grating at the top, the corresponding absorption efficiency decreases significantly. It is further demonstrated that the absorption is enhanced due to the surface plasmon resonance excited by the metal grating in our proposed structure. Through 
the detailed analysis above, it is confirmed that the excellent absorption performance mainly comes from the synergy of surface plasma resonance and the inherent broadband spectral response of the refractory metals.

\section{Conclusion}

In this work, a near-perfect absorber incorporating metal-dielectric-metal subwavelength grating structure with refractory materials is numerically and theoretically analyzed. The average absorption of TMpolarized is $98.4 \%$, and the peak absorption can reach $99.6 \%$. This excellent absorption performances are due to the synergy of surface plasma resonance and the inherent broadband spectral response of refractory metals. The advantage of this structure is that its absorption performance has good tolerance for structural parameters, which can reduce the strict requirements for manufacturing process. The results of this paper can provide reference for the design of absorbers working in the visible with refractory materials, and are expected to find potential applications in structural color, solar-energy harvesting, and other optical devices.

\section{Declarations}

\section{Funding}

The authors acknowledge financial support from the National Natural Science Foundation of China (No. $61505134,61575133,61775076,61405133)$, the Natural Science Foundation of Jiangsu Province (No. BK20140357), the Key Natural Science Foundation of the Higher Education Institutions of Jiangsu Province (No. 14KJB140014), the Science and Technology Project of Suzhou (No. ZXG201427), Jiangsu Planned Projects for Postdoctoral Research Funds (2020Z116) and the project funded by the Priority Academic Program Development of Jiangsu Higher Education Institutions (PAPD).

\section{Conflicts of interest}

The authors have no relevant financial or non-financial interests to disclose.

\section{Ethics approval}

Not applicable.

\section{Consent to participate}

Informed consent was obtained from all individual participants included in the study.

\section{Consent for publication}

Not applicable.

\section{Availability of data and material}


The datasets generated during and/or analysed during the current study are available from the corresponding author on reasonable request.

\section{Code availability}

Not applicable.

\section{Authors' contributions}

All authors contributed to the study conception and design. Material preparation, data collection and analysis were performed by Wenlong Zou, Heng Zhang and Yun Zhou. The first draft of the manuscript was written by Yun Zhou and all authors commented on previous versions of the manuscript. All authors read and approved the final manuscript.

\section{Statements \& Declarations}

The authors have no relevant financial or non-financial interests to disclose.

\section{References}

1. Zang X, Shi C, Chen L, Cai B, Zhu Y, Zhuang S (2015) Ultra-broadband terahertz absorption by exciting the orthogonal diffraction in dumbbell-shaped gratings. Sci Rep 5:8901

2. Ye Y, Jin Y, He S (2010) Omnidirectional, polarization-insensitive and broadband thin absorber in the terahertz regime. J Opt Soc Am B 27 3:498-504

3. He X, Yan S, Ma Q, Zhang Q, Jia P, Wu F, Jiang J (2015) Broadband and polarization-insensitive terahertz absorber based on multilayer metamaterials. Opt Commun 340:44-49

4. Cheng CW, Abbas MN, Chiu CW, Lai KT, Shih MH, Chang YC (2012) Wide-angle polarization independent infrared broadband absorbers based on metallic multi-sized disk arrays. Opt Express 20:10376-10381

5. Cai Y, Zhu J, Liu QH (2015) Enhanced spatial near-infrared modulation of graphene-loaded perfect absorbers using plasmonic nanoslits. Opt Express 23:32318-32328

6. Peng Y, Zang X, Zhu Y (2015) Ultra-broadband terahertz perfect absorber by exciting multi-order diffractions in a double-layered grating structure. Opt Express 23:2032-2039

7. Lin $\mathrm{CH}$, Chen RL, Lin HY (2011) Polarization-independent broad-band nearly perfect absorbers in the visible regime. Opt Express 19:415-424

8. Ji T, Peng L, Zhu Y (2015) Plasmonic broadband absorber by stacking multiple metallic nanoparticle layers. Appl Phy Lett 106:161107

9. Zhou L, Zhou Y, Zhu YF (2016) Broadband bidirectional visible light absorber with wide angular tolerance. J Mater Chem C 4:391-397

10. Qian QY, Sun T, Yan Y, Wang CH (2017) Large-area wide-incident-angle metasurface perfect absorber in total visible band based on coupled Mie resonances. Adv Opt Mater 5:1700064 
11. Zang X, Shi C, Chen L, Cai B, Zhu Y, Zhuang S (2015) Ultra-broadband terahertz absorption by exciting the orthogonal diffraction in dumbbell-shaped gratings. Sci Rep 5:8901

12. He X, Yan S, Ma Q, Zhang Q, Jia P, Wu F, Jiang J (2015) Broadband and polarization-insensitive terahertz absorber based on multilayer metamaterials. Opt Commun 340:44-49

13. Cheng CW, Abbas MN, Chiu CW, Lai KT, Shih MH, Chang YC (2012) Wide-angle polarization independent infrared broadband absorbers based on metallic multi-sized disk arrays. Opt Express 20:10376-10381

14. Ye $Y$, Jin $Y$, He S (2010) Omnidirectional, polarization-insensitive and broadband thin absorber in the terahertz regime. J Opt Soc Am B 27:498-504

15. Peng $Y$, Zang $X$, Zhu $Y$ (2015) Ultra-broadband terahertz perfect absorber by exciting multi-order diffractions in a double-layered grating structure. Opt Express 23:2032-2039

16. Rufangura P, Sabah C (2016) Design and characterization of a dual-band perfect metamaterial absorber for solar cell applications. J Alloys and Compd 671:43-50

17. Zhu B, Huang C, Feng Y, Zhao J, Jiang T (2010) Polarization insensitive metamaterial absorber with wide incident angle. Prog Electromagn Res 101:231-239

18. Huang L, Chen H (2011) Multi-band and polarization insensitive metamaterial absorber. Prog Electromagn Res 113:103-110

19. Batuhan M, Sabah C (2016) Multiband metamaterial absorber design based on plasmonic resonances for solar energy harvesting. Plasmonics 11:1313-1321

20. Wu S, Ye Y, Chen L (2018) A broadband omnidirectional absorber incorporating plasmonic metasurfaces. J Mater Chem C 6:11593-11597

21. Luo M, Zhou Y, Wu S, Chen L (2017) Wide-angle broadband absorber based on one-dimensional metasurface in the visible region. Appl Phys Express 10:092601

22. Tae LK, Ji CG, Guo L (2016) Wide-angle, polarization-independent ultrathin broadband visible absorbers. Appl Phy Lett 108:031107

23. Lei L, Li S, Huang H, Tao K, Xu P (2018) Ultra-broadband absorber from visible to near-infrared using plasmonic metamaterial. Opt Express 26:5686-5693

24. Li Y, Liu Z, Zhang H, Tang P, Wu B, Liu G (2019) Ultra-broadband perfect absorber utilizing refractory materials in metal-insulator composite multilayer stacks. Opt Express 27:11809-11818

25. Li Y, Liu Y, Liu Z, Tang Q, Shi L, Chen Q, Li L (2019) Grating-assisted ultra-narrow multispectral plasmonic resonances for sensing application. Appl Phys Express 12:072002

26. Liu G, Liu X, Chen J, Li Y, Shi L, Fu G, Liu Z (2019) Near-unity, full-spectrum, nanoscale solar absorbers and near-perfect blackbody emitters. Sol Energy Mater Sol Cells 190:20-29

27. Liu Z, Tang P, Liu X, Yi Z, Liu G, Wang Y, Liu M (2019) Truncated titanium/semiconductor cones for wide-band solar absorbers. Nanotechnology 30(30):305203

28. Han S, Lee BJ (2016) Electromagnetic resonance modes on a two-dimensional tandem grating and its application for broadband absorption in the visible spectrum. Opt Express 24:A202-A214 
29. Luo M, Zhou Y, Wu S, Chen L (2017) Wide-angle broadband absorber based on one-dimensional metasurface in the visible region. Appl Phys Express 10:092601

30. Li Y, Liu Z, Zhang H, Tang P, Wu B, Liu G (2019) Ultra-broadband perfect absorber utilizing refractory materials in metal-insulator composite multilayer stacks. Opt Express 27:11809-11818

31. Lin CH, Chen RL, Lin HY (2011) Polarization-independent broad-band nearly perfect absorbers in the visible regime. Opt Express 19:415-424

32. Zhou L, Zhou Y, Zhu YF (2016) Broadband bidirectional visible light absorber with wide angular tolerance. J Mater Chem C 4:391-397

33. Shen S, Tang J, Yu J, Zhou L, Zhou Y (2019) Double-sided and omnidirectional absorption of visible light in tapered dielectric nanostructure coated with non-noble metal. Opt Express 27:24989-24999

34. Zhu P, Guo LJ (2012) High performance broadband absorber in the visible band by engineered dispersion and geometry of a metal-dielectric-metal stack. Appl Phys Lett 101:241116

35. Palik E, Ghosh G (1985)Handbook of Optical Constants of Solids

36. Moharam MG, Grann EB, Pommet DA, Gaylord TK (1995) Formulation for stable and efficient implementation of the rigorous coupled-wave analysis of binary gratings. J Opt Soc Am A 12:10681076

37. Moharam MG, Pommet DA, Grann EB, Gaylord TK (1995) Stable implementation of the rigorous coupled-wave analysis for surface-relief gratings: enhanced transmittance matrix approach. J Opt Soc Am A 12:1077-1086

38. Liang Y, Peng W, Hu R, Xie L (2014) Extraordinary optical properties in the subwavelength metallodielectric free-standing grating. Opt Express 22:19484-19494

39. Abedini Dereshgi S, Ghobadi A, Hajian H, Butun B, Ozbay E (2017) Ultra-broadband, lithography-free, and large-scale compatible perfect absorbers: The optimum choice of metal layers in metal-insulator multilayer stacks. Sci Rep 7:14872

\section{Figures}
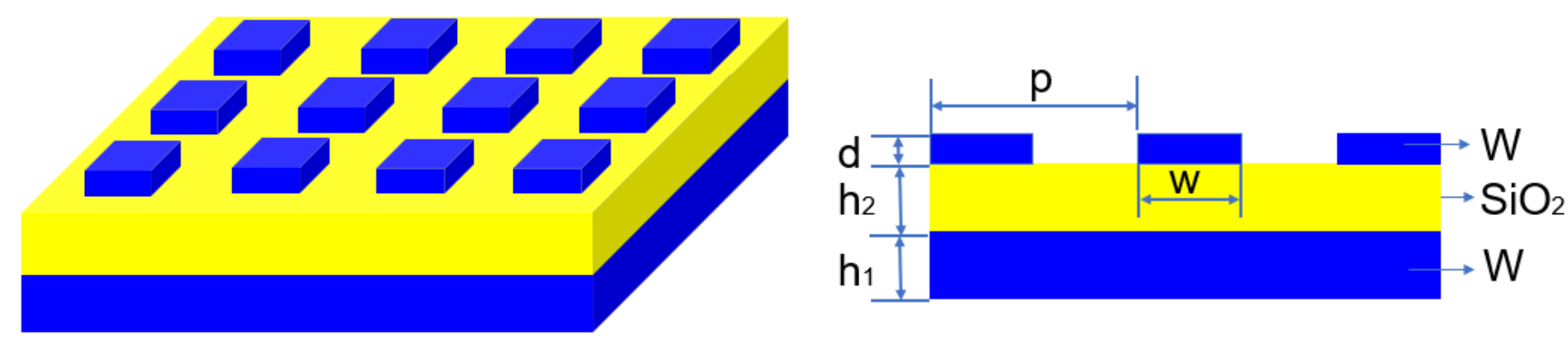

\section{Figure 1}

Schematic diagram and geometric parameters of the proposed absorber. 


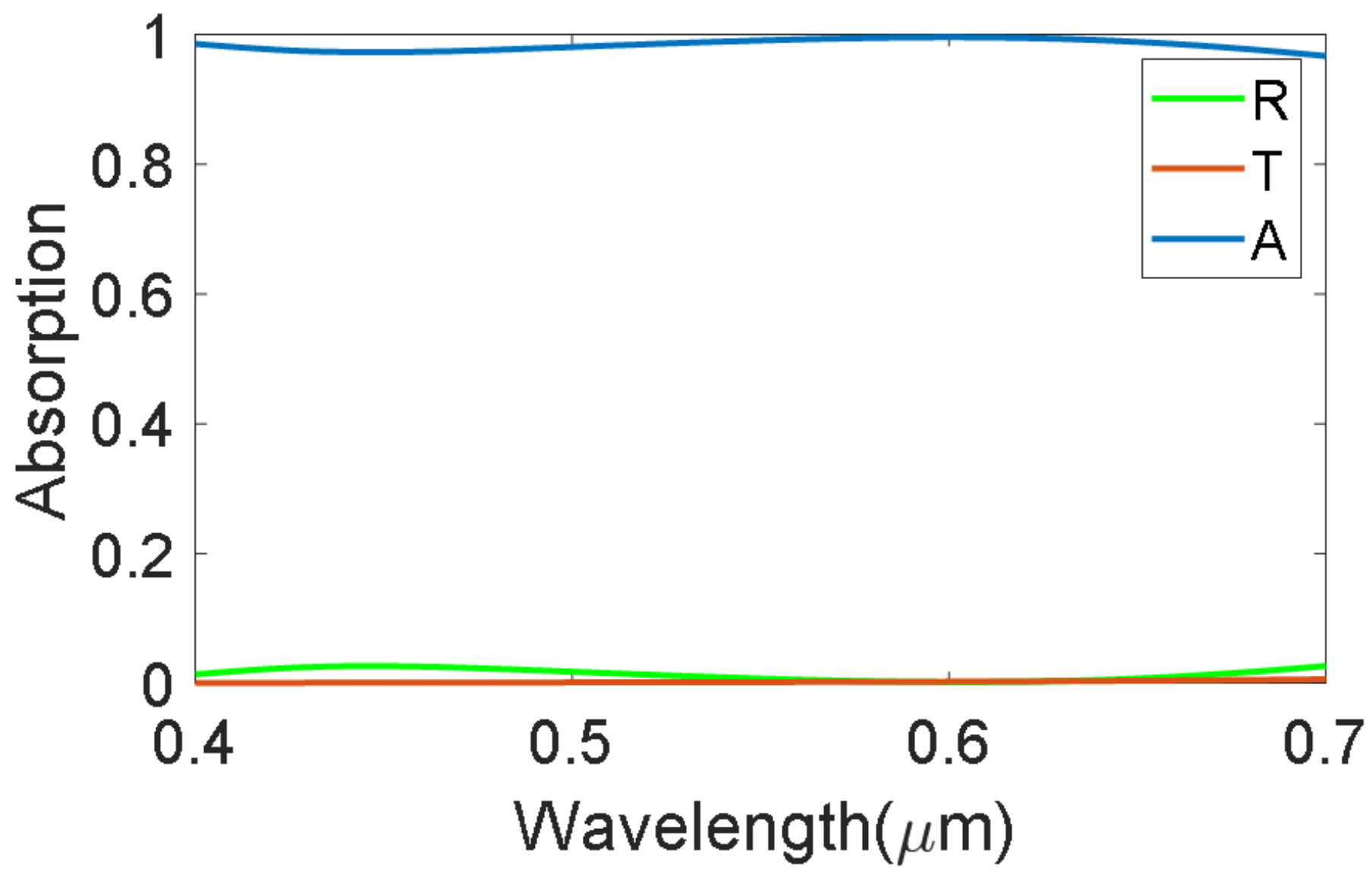

Figure 2

The simulated reflection (R), transmission $(T)$ and absorption (A) spectrum at normal incidence, respectively. 


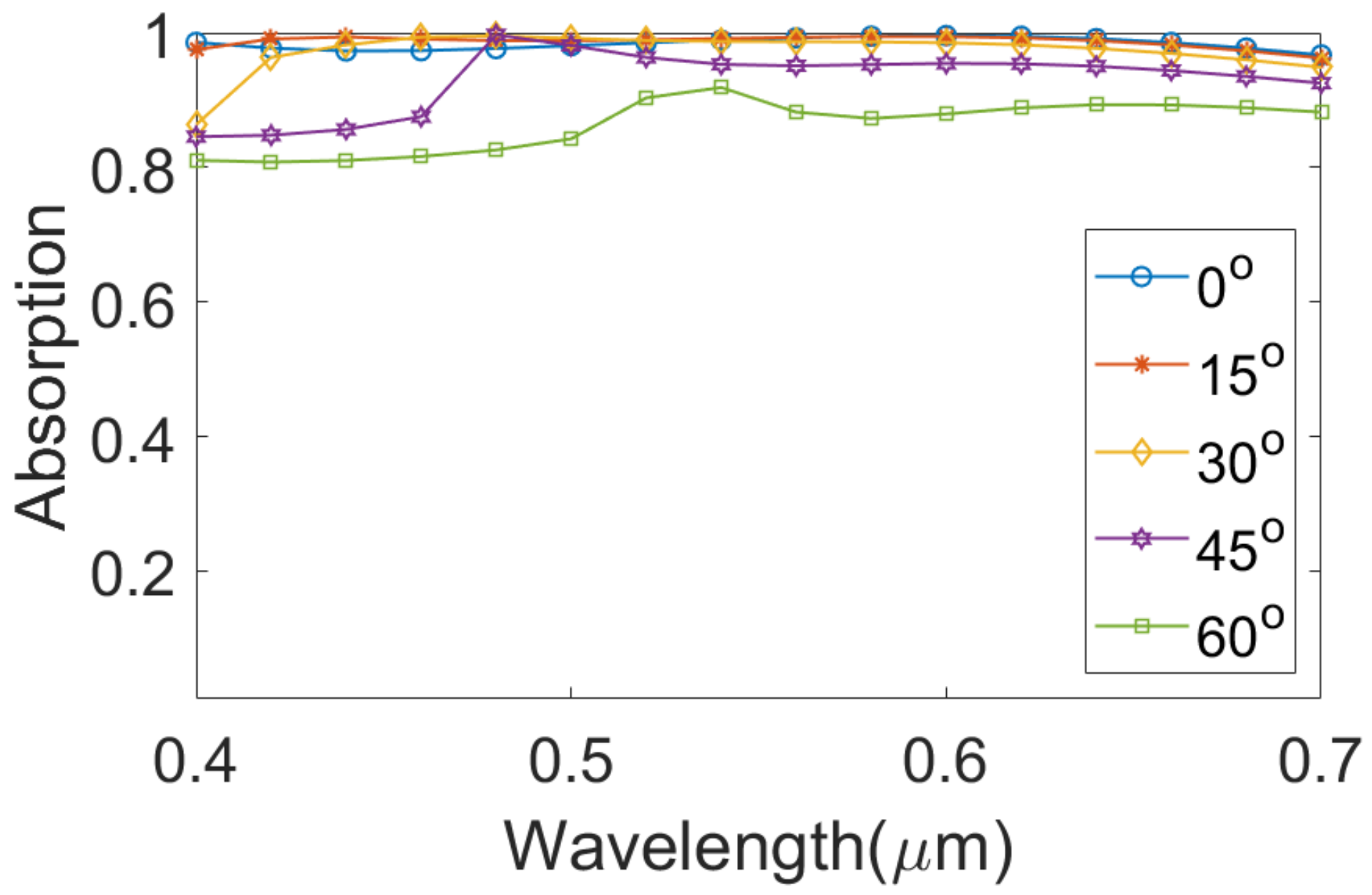

Figure 3

Absorbance spectra as a function of the incident angle for TM-polarized light. 


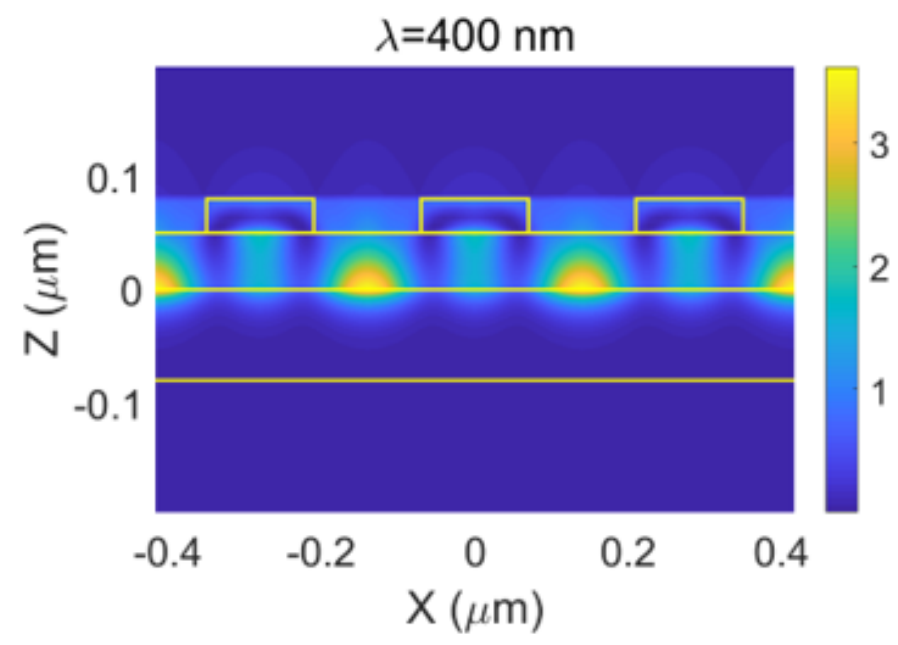

(a)

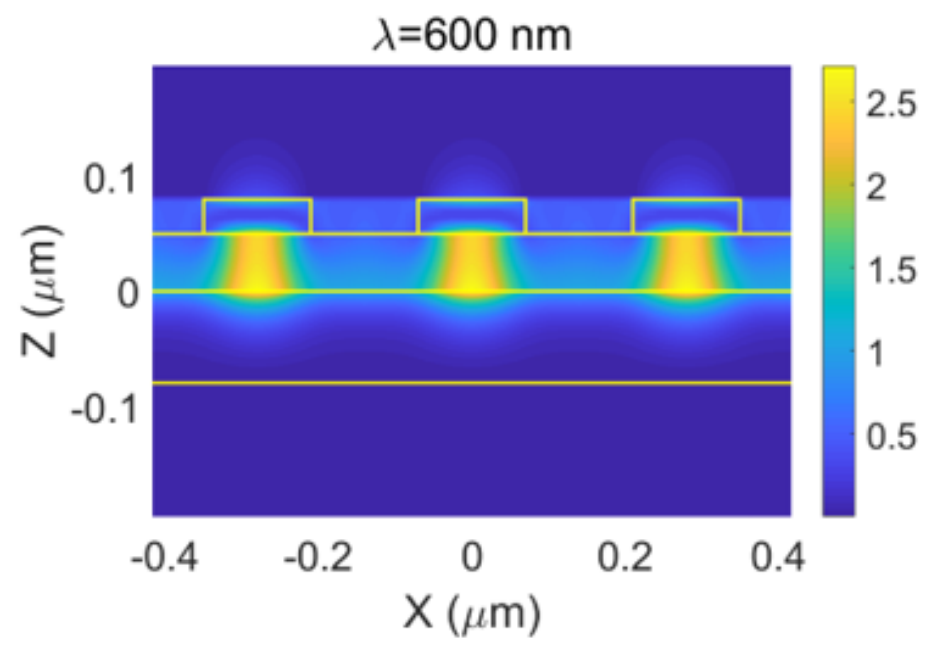

(c)

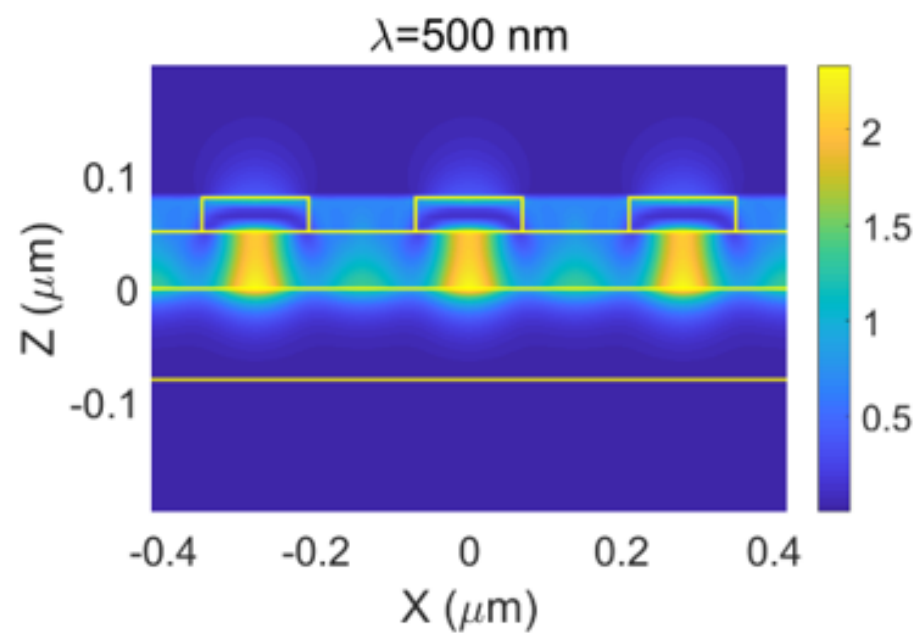

(b)

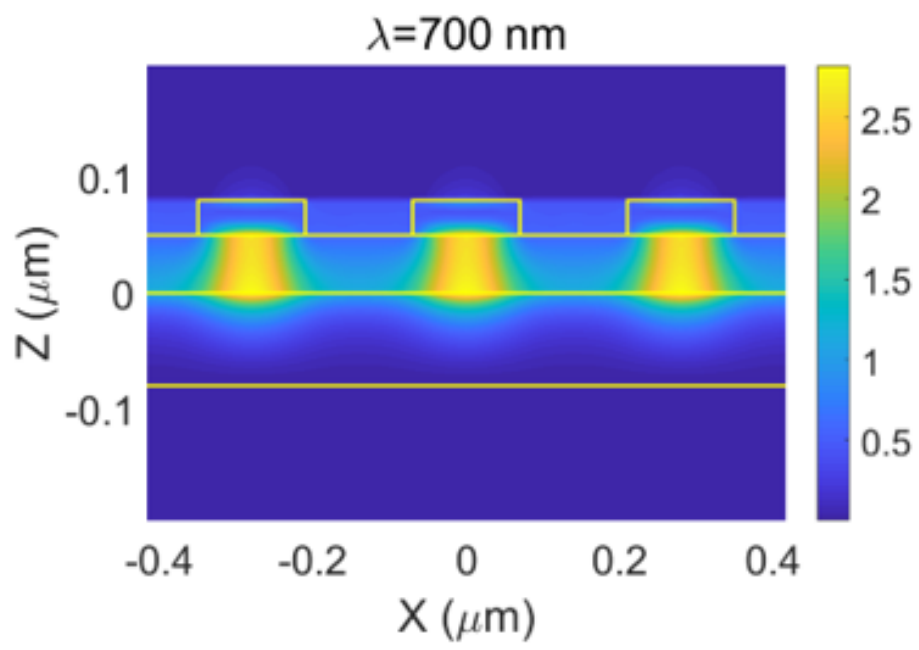

(d)

Figure 4

Calculated magnetic field distributions for TM-polarized light at normal incidence: (a) at $\lambda=400 \mathrm{~nm}$. (b) at $500 \mathrm{~nm}$. (c) at $600 \mathrm{~nm}$. (d) at $700 \mathrm{~nm}$. 


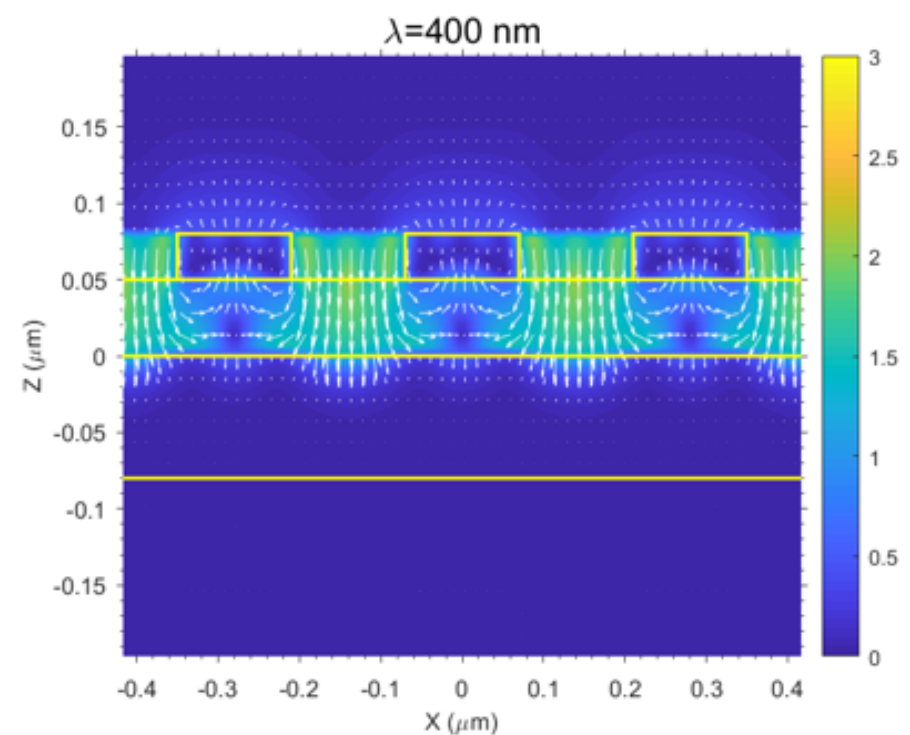

(a)

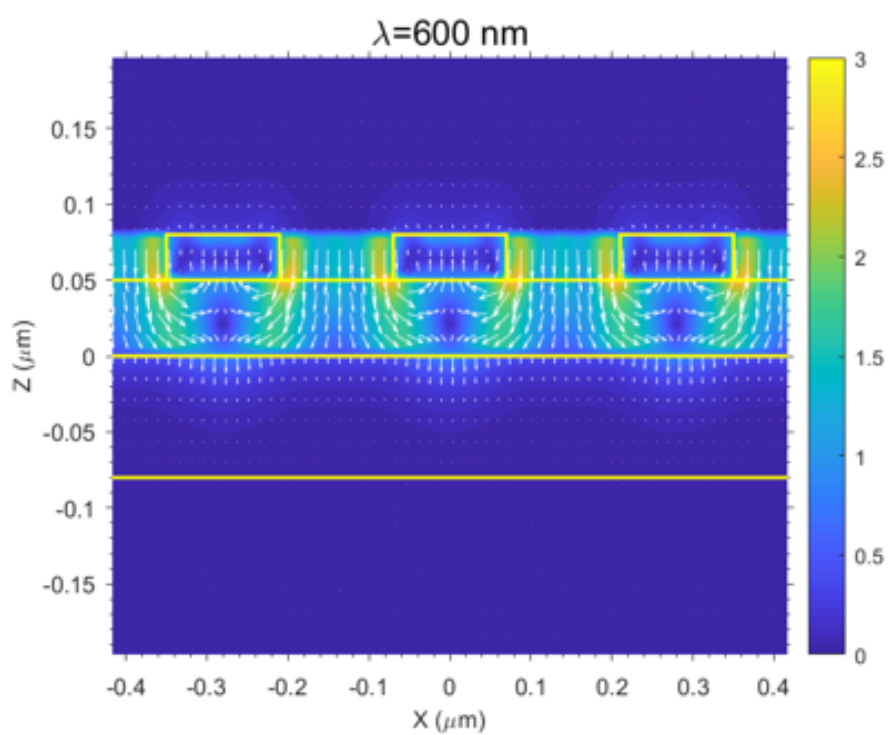

(b)

\section{Figure 5}

Calculated the Poynting vector distributions for TM-polarized light at normal incidence: (a) at $\lambda=400 \mathrm{~nm}$. (b) at $600 \mathrm{~nm}$. 


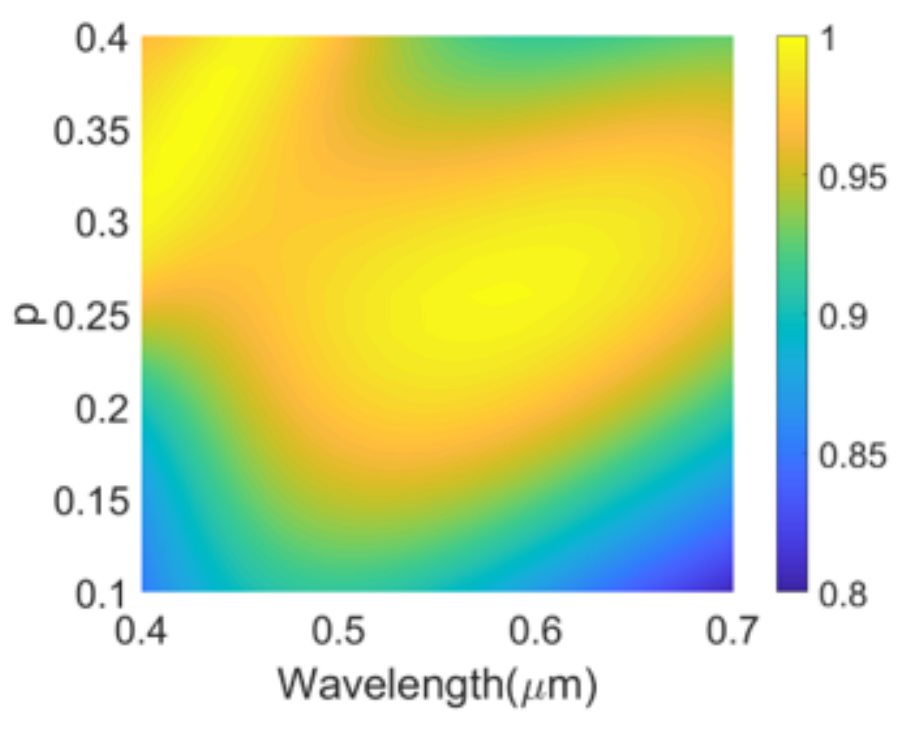

(a)

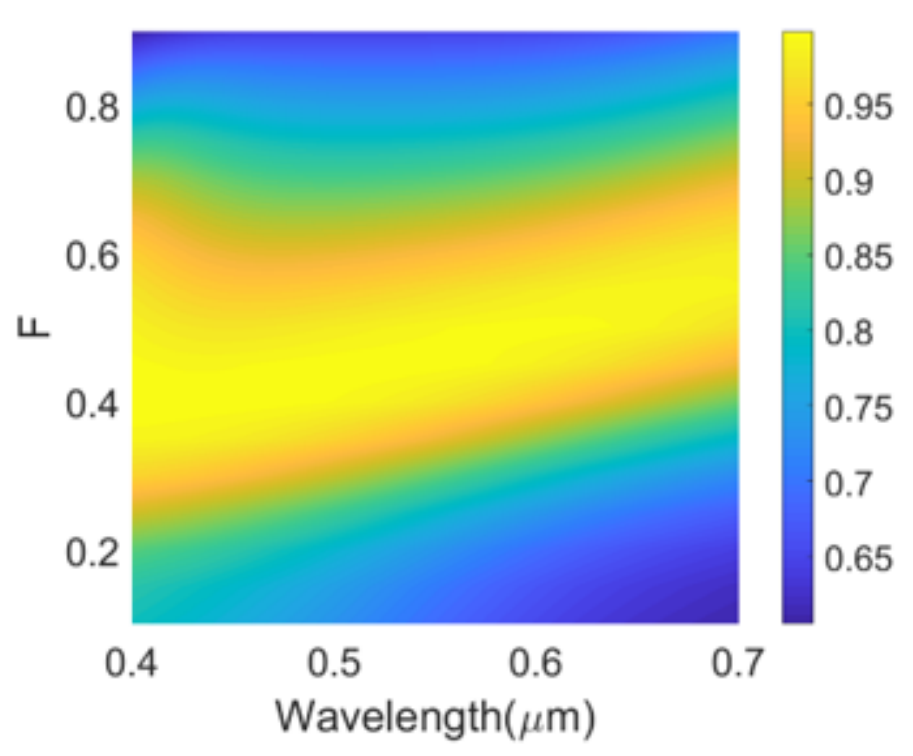

(c)

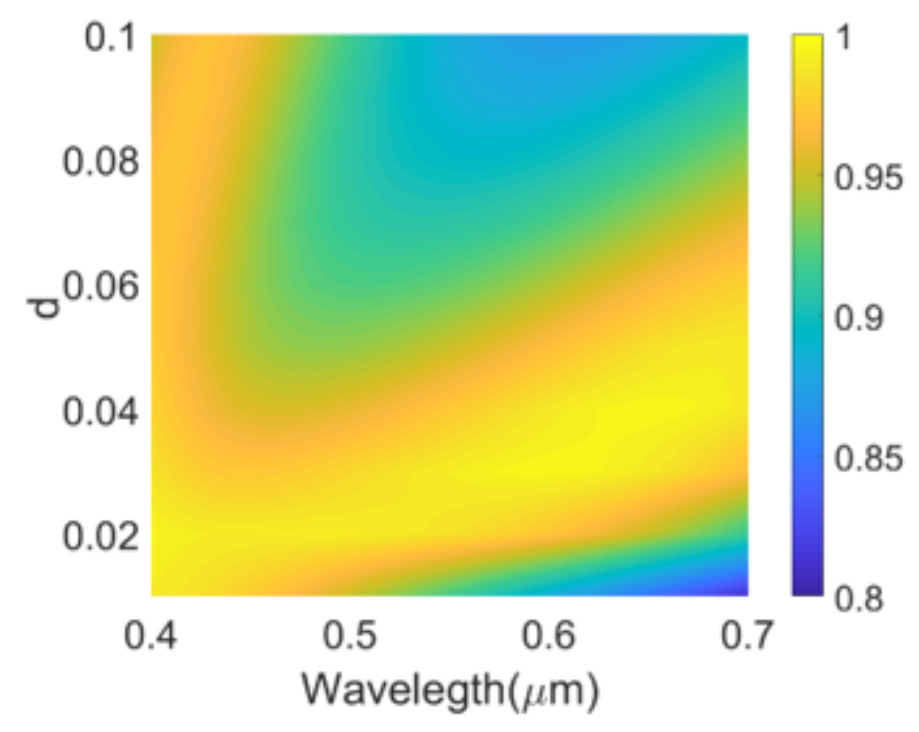

(b)

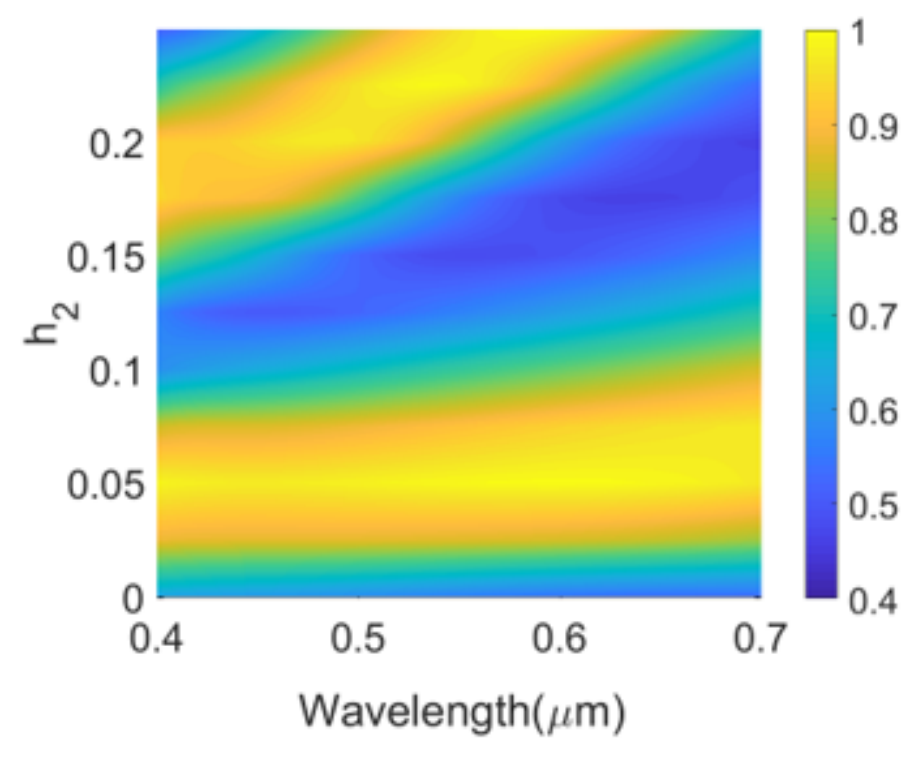

(d)

\section{Figure 6}

Dependence of the absorption on the parameters of the proposed absorber: (a) the period of the metal grating (p). (b) the height of the metal grating (d). (c) the duty cycle of the metal grating (F). (d) the height of the dielectric layer $\left(\mathrm{h}_{2}\right)$. 


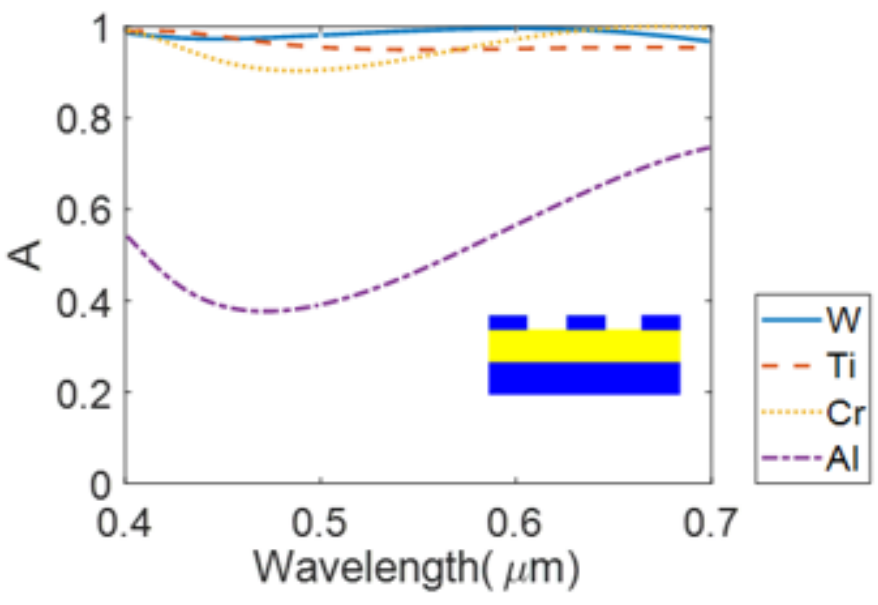

(a)

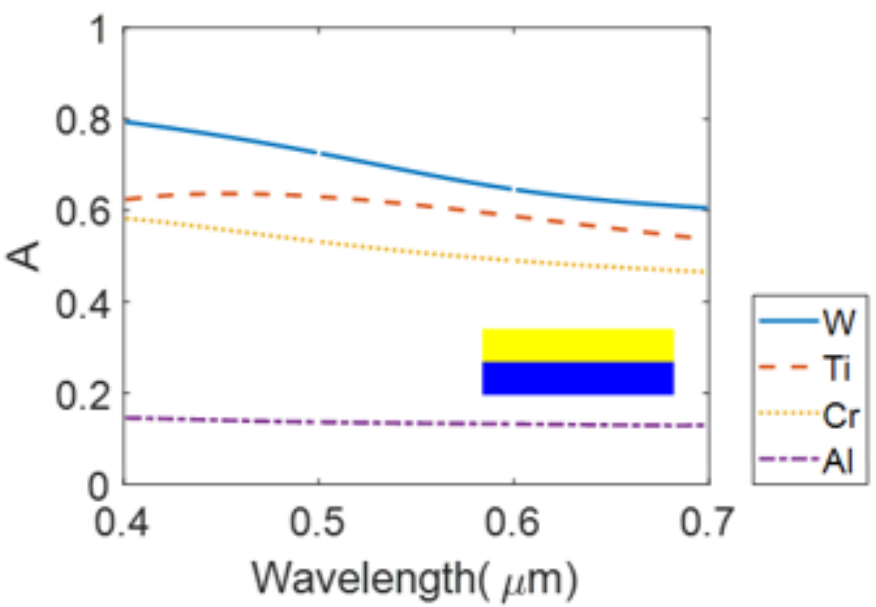

(b)

Figure 7

Absorption spectra using metals of $\mathrm{W}, \mathrm{T}, \mathrm{Cr}$ and $\mathrm{Al}$. 